\title{
GDFII restrains tumor growth by promoting apoptosis in pancreatic cancer
}

This article was published in the following Dove Press journal:

OncoTargets and Therapy

\author{
Yanzhe Liu',* \\ Lijuan Shao ${ }^{2, *}$ \\ Kuang Chen' \\ Zizheng Wang' \\ Jin Wang' \\ Wei Jing ${ }^{3}$ \\ Minggen $\mathrm{Hu}^{\prime}$ \\ 'Department of Hepatobiliary \\ and Pancreatic Surgical Oncology, \\ Chinese PLA General Hospital, \\ Beijing, People's Republic of China; \\ ${ }^{2}$ Translational Medicine Collaborative \\ Innovation Center, The Second \\ Clinical Medical College (Shenzhen \\ People's Hospital), Jinan University, \\ Shenzhen, People's Republic of China; \\ ${ }^{3}$ Department of General Surgery, \\ Changhai Hospital, The Second \\ Military Medical University, Shanghai, \\ People's Republic of China \\ *These authors contributed equally \\ to this work
}

Correspondence: Minggen Hu Department of Hepatobiliary and Pancreatic Surgical Oncology, Chinese PLA General Hospital, 28 Fuxing Road, Haidian District, Beijing 100853, People's Republic of China

Email hmg30I@I26.com

Wei Jing

Department of General Surgery, Changhai Hospital, The Second Military Medical University, 800 Xiangyin Road, Shanghai 200040, People's Republic of China

Email jingwei7777@163.com
Background: Growth differentiation factor (GDF) acted as a factor that regulated proliferation, apoptosis and differentiation in several tumors. However, the effects of growth differentiation factor (GDF11) in pancreatic cancer remain unclear.

Purpose: To investigate the expression and significance of GDF11 in pancreatic cancer.

Patients and methods: Pancreatic cancer and corresponding paracancerous tissues $(n=28)$ were collected from the Department of Hepatobiliary and Pancreatic Surgical Oncology of Chinese PLA General Hospital. Tissue microarray was obtained from Outdo Biotech Co., Ltd. (Shanghai, People's Republic of China). GDF11 mRNA and protein expressions in pancreatic cancer samples and cell lines were detected using qRT-PCR, Western-Blot and immunohistochemistry. Overexpression and knockdown of GDF11 were performed with lentiviral transduction system and siRNA technique in PANC-1 cell line and CFPAC-1 cell line. Proliferation, migration and invasion of pancreatic cancer cell lines were examinated by MTS and transwell assay, respectively. Flow cytometry was used for cell apoptosis analysis.

Results: The results of this study indicated that GDF11 was significantly down-regulated in pancreatic cancer tissues compared with adjacent tissues of pancreatic cancer. GDF11 was also associated with low expression in pancreatic cancer cell lines when compared with normal pancreatic cell line. In a cohort of 63 pancreatic cancer patients, high GDF11 expression levels was associated with favorable perineural invasion, $\mathrm{T}$ classification, $\mathrm{N}$ classification and overall survival (OS). Cox proportional hazards model revealed that high GDF11 expression was an independent predictor of favorable prognosis (HR: 0.496; 95\% CI: 0.255-0.967; $P=0.040$ ). Overexpression of GDF11 in PANC-1 cells repressed the proliferation, migration and invasion abilities in vitro. Inhibition of GDF11 in CFPAC-1 showed inverse results. Furthermore, enhanced GDF11 expression promoted apoptosis and down-regulated GDF11 expression inhibited apoptosis in pancreatic cancer cell lines.

Conclusion: These findings suggested that GDF11 acted as a tumor suppressor gene for pancreatic cancer.

Keywords: GDF11, pancreatic cancer, proliferation, apoptosis, prognosis

\section{Introduction}

Pancreatic cancer is a digestive system tumor with high degree of malignancy and poor prognosis, accounting for the sixth leading cause of cancer mortality rate in People's Republic of China. ${ }^{1,2}$ The incidence and mortality rates of pancreatic cancer show a sustained increase in the world, with the overall 5-year survival rate less than $8 \%{ }^{3}$ Its low 5-year survival rate is mainly due to the early metastasis to regional lymph nodes and terminal hematogenous metastasis to distant organs. ${ }^{4}$ Lack of early observable symptoms, higher staging at the time of diagnosis, and few effective therapies are largely responsible for the poor prognosis of pancreatic cancer. Recently, more 
researches have focused on the tumor biomarkers that were associated with pancreatic cancer prognosis., 5 Therefore, it is imperative to identify novel prognostic molecules for improving the outcomes of pancreatic cancer patients.

The growth differentiation factor 11 (GDF11), also called bone morphogenetic protein 11 (BMP11), is a secreted protein in TGF- $\beta$ superfamily and BMP subfamily, which regulates diverse developmental processes from anterior/posterior patterning to myogenesis. ${ }^{7,8}$ It is closely regulated to a negative regulator of muscle growth, GDF8 (myostatin). ${ }^{9}$ With regard to cancer, BMP acted as a factor that regulated cell proliferation, apoptosis, and differentiation ${ }^{10-12}$ and was also shown to be correlated with various cancers such as esophageal cancer, ${ }^{13}$ prostate cancer, ${ }^{14}$ and breast cancer. ${ }^{15}$ GDF11 has been measured in different tissues, such as the pancreas, intestine, kidney, skeletal muscle, heart and developing nervous system, olfactory system, and retina. ${ }^{16}$ Abnormal expression of GDF11 has been found in colorectal carcinoma, and high GDF11 expression was related to poorer prognosis. ${ }^{17}$ A recent study has demonstrated that GDF11 showed tumor-suppressor function in triple-negative breast cancer. ${ }^{18}$ However, to our knowledge, there is no information regarding GDF11 in pancreatic cancer, and the biological function of GDF11 in cancer cells remains largely unknown.

In this study, we first examined the expression of GDF11 in pancreatic cancer. We identified GDF11 as a novel suppressor of pancreatic cancer growth and metastasis through in vitro studies. We also evaluated its association with clinicopathological characteristics and then assessed its prognostic value in pancreatic cancer patients.

\section{Patients and methods}

\section{Patients}

This study was conducted after approval by the Ethic Committee of Chinese PLA General Hospital, and written informed consent was obtained from each patient. Tissue samples including tumor tissues and adjacent tissues of cancer were obtained from a total of 28 patients who had undergone surgical resection and confirmed the diagnosis of pancreatic cancer by postoperative pathology between December 2016 and March 2017 in Chinese PLA General Hospital, Beijing, People's Republic of China. All the tissues were put into liquid nitrogen for frozen immediately after resection and stored at $-80^{\circ} \mathrm{C}$ for RT-PCR analysis and Western blot.

\section{Cell lines and culture}

Pancreatic cancer cell lines AsPC-1, BxPC-3, CFPAC-1, PANC-1, and SW1990 and human pancreatic duct epithelial cell line HPDE6-C7 were purchased from China
Infrastructure of Cell Line Resources (Beijing, People's Republic of China) and cultured in Dulbecco's Modified Eagle's Medium (Hyclone Co., Logan, UT, USA) and supplemented with $10 \% \mathrm{FBS}$ (Hyclone Co.) at $37^{\circ} \mathrm{C}$ in a humidified $5 \% \mathrm{CO}_{2}$ air incubator.

\section{RNA extraction and qRT-PCR}

Total RNA was extracted from fresh tumor tissues and adjacent tissues of cancer of 28 patients using TRIzol reagent (Qiagen, Hilden, Germany) and was reverse-transcribed using a High Capacity cDNA Reverse Transcription kit (TaKaRa, Kusatsu, Japan). Levels of the corresponding peptidyl prolyl isomerase A (PPIA) and GDF11 mRNA were detected by qRT-PCR using the ABI7500 RealTime PCR System (Thermo Fisher Scientific, Waltham, MA, USA). Housekeeping gene PPIA was used as internal standard for GDF11 mRNA. The sequences of the qRT-PCR primer were as follows: GDF11 forward, 5'-GATCTTGAGCAAACTGCGGC-3'; GDF11 reverse, 5'-TGAAGTCGATGCTCTGCCAA-3'; PPIA forward, 5'-TCATCTGCACTGCCAAGACTG-3; PPIA reverse, 5'-CATGCCTTCTTTCAC TTTGCC-3'.

\section{Western blot analysis}

Five pancreatic cancer cell lines, a human pancreatic duct epithelial cell line, eight samples of pancreatic cancer tissue and paired nontumor tissue were used for Western blot analysis. Proteins were extracted from cells and tissues using RIPA lysis buffer with proteinase inhibitor. Then, proteins were separated by SDS-PAGE and transferred to polyvinylidene fluoride (PVDF) membranes. The membranes were blocked with 5\% nonfat milk and $0.01 \%$ Tween-20 in tris-buffered saline (TBS; $\mathrm{pH} 7.6$ ) and incubated with GDF11 antibody (Santa Cruz Biotechnology Inc., Dallas, TX, USA) diluted at 1:200 in TBS overnight at $4^{\circ} \mathrm{C}$. Subsequently, a 1:5,000 dilution of the goat antimouse IgG alkaline phosphatase-linked secondary antibody (Sigma-Aldrich Co., St Louis, MO, USA) was incubated at room temperature. The antigen-antibody complex was visualized by Immobilon ${ }^{\mathrm{TM}}$ Western Chemiluminescence HRP Substrate (EMD Millipore, Billerica, MA, USA).

\section{Tissue microarray and immunohistochemistry (IHC)}

Tissue microarray was obtained from Outdo Biotech Co., Ltd. (Shanghai, People's Republic of China). IHC studies of GDF11 were performed on pancreatic cancer samples of tissue microarray. IHC was performed as previously described. ${ }^{19}$ Rabbit antihuman polyclonal GDF11 
antibody (Abcam Co., Cambridge, MA, USA) was used at a 1:500 dilution. Quantitative analysis of the staining was performed based on the percentage of positive cells and staining density by three experienced pathologists using 12 standard points. For data analysis, staining scores $<9$ were defined as low expression, and scores of 9-12 indicated high expression.

\section{Flow cytometry}

For cell apoptosis analysis, $1 \times 10^{6}$ cells in PBS were stained with FITC-Annexin V and PI and incubated in the dark at room temperature. Annexin V/PI staining assays were performed following the manufacturer's protocol (BD Biosciences, San Jose, CA, USA), and the samples were assessed using flow cytometry. Data acquisition and analysis were performed on an FC500 MPL system (Beckman Coulter, Inc., Brea, CA, USA).

\section{Plasmid construction}

V5-tagged GDF11 was constructed by cloning the fulllength cDNA into pLV-sfGFP-Puro vectors, yielding lentiviral vectors (LV)-GDF11. Empty vector with no cDNA fragment was used as the negative control (NC), termed LV-Control.

\section{RNAi treatment}

siRNAs targeting GDF11 and related NC were chemically synthesized by GenePharma (Shanghai, People's Republic of China). The sequences of the GDF11 siRNA are as follows: 5'-CCGAGACCGUCAUUAGCAUTT-3' (sense) and 5'-AUGCUAAUGACGGUCUCGGTT-3' (antisense). The sequences of siNC are as follows:

5'-UUCUCCGAACGUGUCACGUTT-3' (sense) and 5'-ACGUGACACGUUCGGAGAATT-3' (antisense). Transfection was carried out using Lipofectamine ${ }^{\circledR} 2000$ (Invitrogen, Carlsbad, CA, USA) in accordance with the manufacturer's protocol.

\section{3-(4,5-dimethylthiazol-2-yl)-5-} (3-carboxymethoxyphenyl)-2-(4sulfophenyl)-2H-tetrazolium (MTS) assay

Cell growth was measured by MTS assays (Promega, Madison, WI, USA) in 96-well plates (1,000 cells per well) following manufacturer instructions.

\section{Transwell migration and invasion assay}

Cells in medium with no FBS were seeded into upper chambers of Matrigel-coated ( $8 \mu \mathrm{m}$ pore size; HTSTM Fluoroblok invasion system, BD Biosciences) or -uncoated polycarbonate membrane filters $(8 \mu \mathrm{m}$ pore size; Corning
Incorporated, Corning, NY, USA). The lower chamber was filled with medium containing 10\% FBS. Then, cells were incubated in $37^{\circ} \mathrm{C}$. After 24 and 48 hours, cells that migrated through the membrane and attached to the bottom of the membrane were fixed and stained with $0.05 \%$ crystal violet for 30 minutes. Images were captured from five random fields under microscope. All experiments were performed in triplicate.

\section{Statistical analysis}

SPSS 22.0 software (SPSS Inc., Chicago, IL, USA) was used for the statistical analysis. Data were expressed as the mean \pm standard deviation (SD). Student's $t$-test was used to compare data between two groups. Pearson chisquared test or Fisher's exact test was used to analyze the relationship between GDF11 expression and clinicopathological characteristics. Kaplan-Meier analysis was performed to assess the differences in survival rates. Each test was two sided, and $P<0.05$ was considered statistically significant.

\section{Results \\ GDFI I mRNA and protein expressions in pancreatic cancer samples and cell lines}

To validate the protein and mRNA expression level of GDF11, 28 pairs of tissues including pancreatic cancer samples and matched paracarcinoma tissue samples were detected by qRT-PCR and eight pairs were detected by Western blot in this study. The mRNA and protein levels of GDF11 were decreased in pancreatic cancer samples compared to normal tissues (Figure 1A and B). Western blot and qRT-PCR analyses revealed that GDF11 expression was downregulated in pancreatic cancer cell lines than HPDE6-C7 cell line (Figure 1C and D). Moreover, IHC staining of 28 pairs of pancreatic adenocarcinoma tissues and normal pancreas tissues revealed that GDF11 was localized in cytoplasm. High-level expression of GDF11 was detected in $24 / 28(85.7 \%)$ of adjacent paracarcinoma tissues, but only $10 / 28(35.7 \%)$ in cancerous tissues (Figure 1E). The results acquired demonstrated that GDF11 might serve as a tumor suppressor in pancreatic cancer.

\section{The association between GDFI I expression and clinicopathological characteristics}

The correlation of GDF11 expression with clinicopathological characteristics in tissue microarray of 63 pancreatic cancer patients is further summarized in Table 1. Same as above, 
A

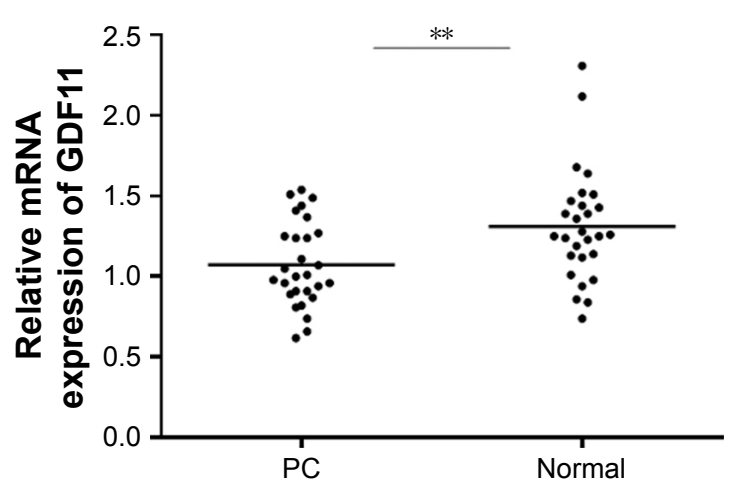

C

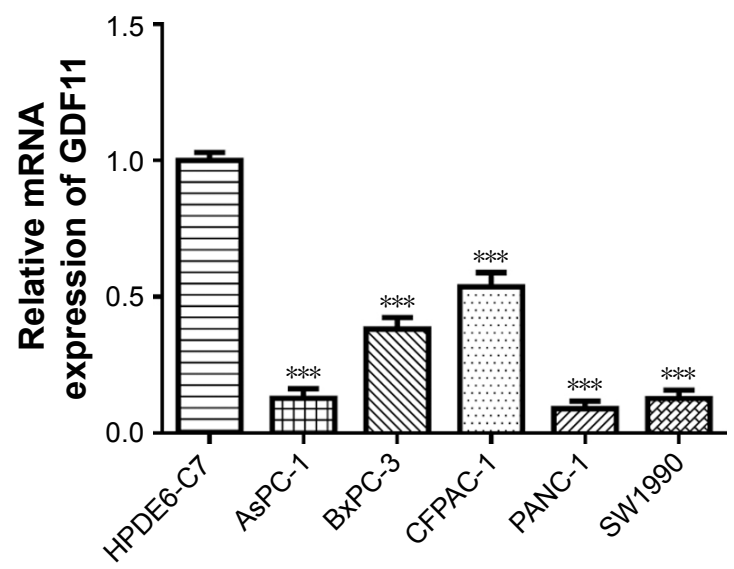

E

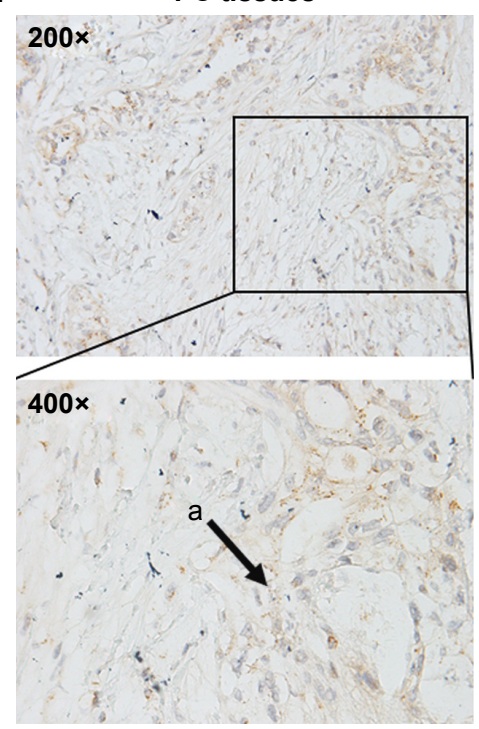

B

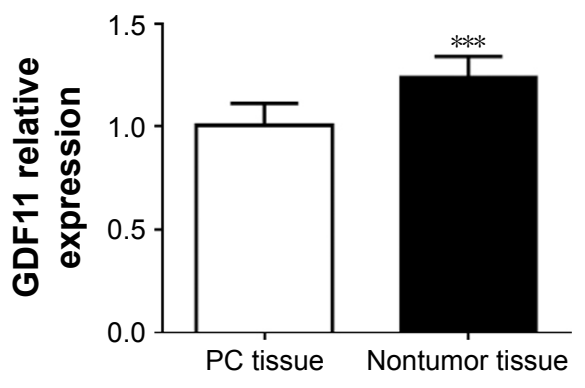

$$
\frac{1}{T N} \frac{2}{T N} \frac{3}{T N} \frac{4}{T N} \frac{5}{T N} \frac{6}{T N} \frac{7}{T N} \frac{8}{T N}
$$

GDF11 - - - - - - - - - - - - - - -

GAPDH

D

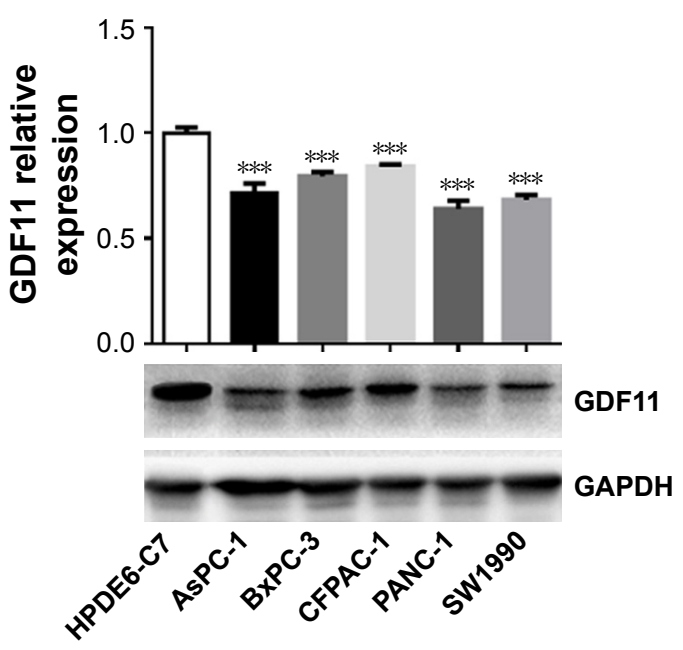

Normal pancreatic tissues

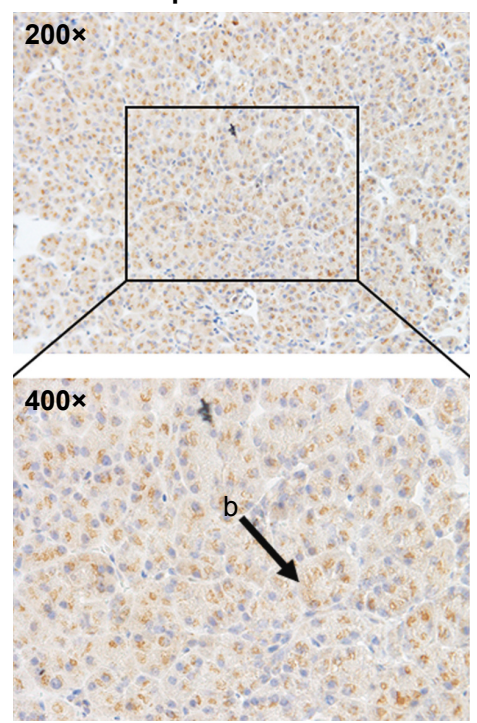

Figure I The mRNA and protein expression of GDFII in pancreatic cancer cell lines and clinical samples.

Notes: (A) qRT-PCR analysis of GDFII levels in pancreatic cancer tissues and normal pancreatic tissues ( $\mathrm{n}=28$ ). (B) Protein levels of GDFII were detected by Western blot in eight pairs of pancreatic cancer tissues and normal pancreatic tissues. (C) The mRNA expressions of GDFII in the normal pancreatic cell line HPDE6-C7 and five pancreatic cancer cell lines were examined by qRT-PCR. (D) Western blot analysis of GDFII in the HPDE6-C7 and five pancreatic cancer cell lines. (E) Representative images and its regional magnification of GDFII IHC in pancreatic cancer tissues and their paired normal tissues. a: Low expression of GDFII in pancreatic cancer tissues; b: high expression of GDFII in the cytoplasm of normal pancreatic tissues. Data shown represent the mean $\pm S D$. $* * P<0.01$. $* * * P<0.001$.

Abbreviations: GDFI I, growth differentiation factor II; IHC, immunohistochemistry; PC, pancreatic cancer; GAPDH, glyceraldehyde-3-phosphate dehydrogenase. 
Table I The relationship between GDFII expression and clinicopathological characteristics in tissue microarray of 63 pancreatic cancer patients

\begin{tabular}{|c|c|c|c|c|}
\hline \multirow[t]{2}{*}{ Characteristics } & \multirow[t]{2}{*}{$\mathbf{N}$} & \multicolumn{2}{|l|}{ GDFI I } & \multirow[t]{2}{*}{$P$-value } \\
\hline & & $\begin{array}{l}\text { High } \\
\text { expression }\end{array}$ & $\begin{array}{l}\text { Low } \\
\text { expression }\end{array}$ & \\
\hline Gender & & & & 0.212 \\
\hline Male & 36 & 13 & 23 & \\
\hline Female & 27 & 14 & 13 & \\
\hline Age (years) & & & & 0.229 \\
\hline$\leq 65$ & 12 & 7 & 5 & \\
\hline$>65$ & 51 & 20 & 31 & \\
\hline Differentiation & & & & $0.94 I$ \\
\hline Well/moderate & 37 & 16 & 21 & \\
\hline Poor & 26 & 11 & 15 & \\
\hline Perineural invasion & & & & 0.019 \\
\hline No & 36 & 20 & 16 & \\
\hline Yes & 27 & 7 & 20 & \\
\hline T classification & & & & 0.010 \\
\hline $\mathrm{TI}-\mathrm{T} 2$ & 28 & 17 & 11 & \\
\hline T3-T4 & 35 & 10 & 25 & \\
\hline $\mathrm{N}$ classification & & & & 0.029 \\
\hline No & 32 & 18 & 14 & \\
\hline NI & 31 & 9 & 22 & \\
\hline
\end{tabular}

Abbreviation: GDFI I, growth differentiation factor II.

IHC staining showed that the high-level expression rate of GDF11 was lower in cancerous tissues than that in normal tissues (Figure 2A). Kaplan-Meier method for overall survival (OS) indicated that the patients with high GDF11 expression had significantly better survival rates compared to patients with low GDF11 expression (Figure 2B). Univariate analysis revealed that GDF11 expression $(P=0.001)$, differentiation
( $P=0.026), \mathrm{T}$ classification $(P=0.024)$, and $\mathrm{N}$ classification $(P<0.001)$ were significant prognostic factors for OS. Multivariate analysis further indicated high GDF11 expression as an independent biomarker of favorable prognosis (HR: 0.496; 95\% CI: 0.255-0.967; $P=0.040$ ). However, age, gender, and perineural invasion were not associated with OS (Table 2).

\section{Overexpression of GDFI I suppressed proliferation, migration, and invasion while knockdown of GDFI I facilitated these functions in pancreatic cancer cell lines}

Lentiviral transduction system was used to elevate the expression level of GDF11 in PANC-1 cell line and study biological functions of GDF11 in pancreatic cancer cell. Moreover, we knocked down GDF11 expression using the siRNA technique in CFPAC-1 cell line for same functional experiments. Overexpression and knockdown of GDF11 were confirmed by qRT-PCR and Western blot (Figure 3A). Enhanced GDF11 expression suppressed cell proliferation in PANC-1 cells while CFPAC-1 cell transfected with GDF11-siRNA showed boosted tumor growth (Figure 3B). In addition, migration and invasion assays showed a decrease of cell migration and invasion in group with high GDF11 expression, and these functions were accelerated in GDF11 downregulated group (Figure $3 \mathrm{C}$ and D). These results suggest that GDF11 might act as a tumor suppressor and inhibit the proliferation, migration, and invasion of pancreatic cancer cells.
A

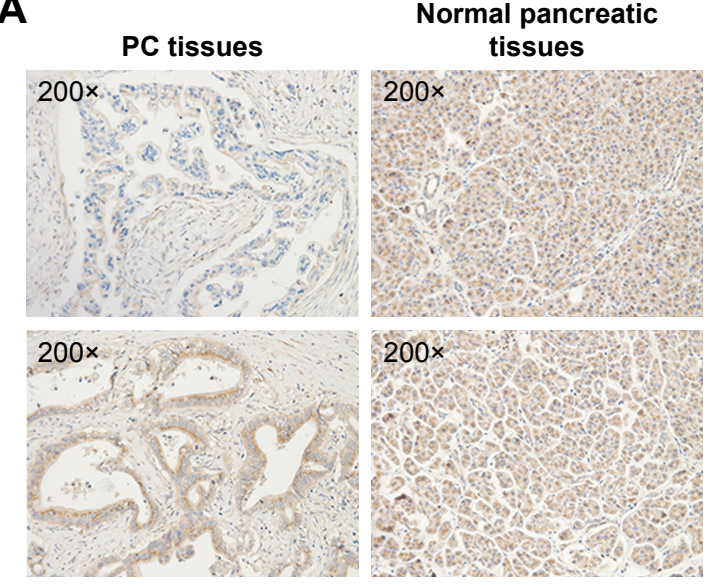

B

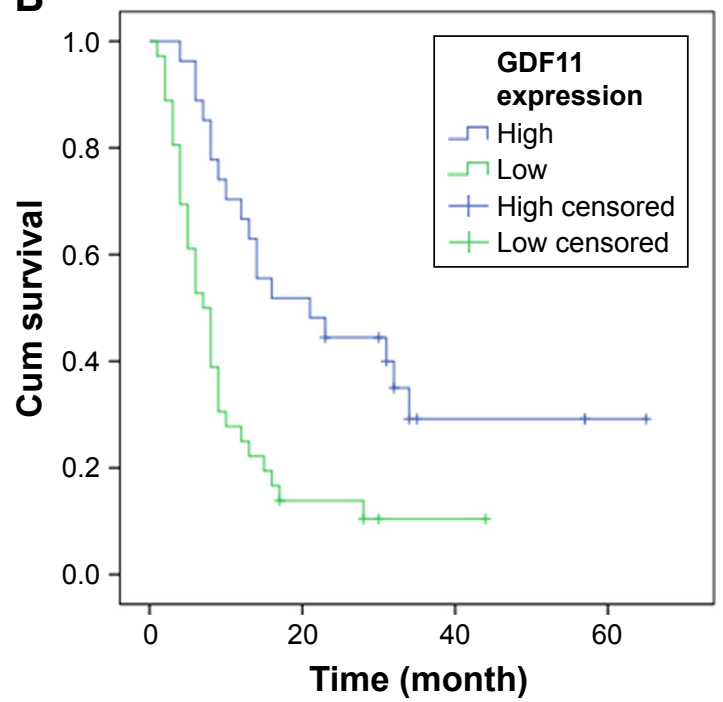

Figure 2 Relationship between GDFII expression and OS in tissue microarray of pancreatic cancer.

Notes: (A) Representative IHC staining images of GDFII of paired tumor and nontumor tissues. (B) Kaplan-Meier analysis showed that patients with low expression of GDFI I had a poorer OS than those with high expression of GDFI I. P=0.012, log-rank test.

Abbreviations: GDFI I, growth differentiation factor II; IHC, immunohistochemistry; OS, overall survival; PC, pancreatic cancer. 
Table 2 Univariate and multivariate Cox regression analyses of factors associated with overall survival

\begin{tabular}{|c|c|c|c|c|c|c|}
\hline \multirow[t]{2}{*}{ Variables } & \multicolumn{3}{|c|}{ Univariate analysis } & \multicolumn{3}{|c|}{ Multivariate analysis } \\
\hline & $P$-value & HR & $95 \% \mathrm{Cl}$ & $P$-value & HR & $95 \% \mathrm{Cl}$ \\
\hline $\begin{array}{l}\text { Gender } \\
\text { (female vs male) }\end{array}$ & 0.507 & 0.826 & $0.470-1.452$ & & & \\
\hline $\begin{array}{l}\text { Age (years) } \\
(>65 \text { vs } \leq 65)\end{array}$ & 0.835 & 1.076 & $0.537-2.156$ & & & \\
\hline $\begin{array}{l}\text { Differentiation } \\
\text { (poor vs well/moderate) }\end{array}$ & 0.026 & 1.898 & $1.080-3.336$ & 0.115 & 1.597 & $0.892-2.862$ \\
\hline $\begin{array}{l}\text { Perineural invasion } \\
\text { (yes vs no) }\end{array}$ & 0.335 & 0.757 & $0.429-1.334$ & & & \\
\hline $\begin{array}{l}\text { T classification } \\
(\text { T3-T4 vs TI-T2) }\end{array}$ & 0.024 & 1.922 & $1.088-3.395$ & 0.067 & 1.748 & $0.961-3.181$ \\
\hline $\begin{array}{l}\text { N classification } \\
(\mathrm{NI} \text { vs } \mathrm{N} 0)\end{array}$ & $<0.001$ & 3.256 & $1.793-5.914$ & 0.005 & 2.544 & $1.325-4.882$ \\
\hline $\begin{array}{l}\text { GDFII } \\
\text { (high vs low) }\end{array}$ & 0.001 & 0.364 & $0.200-0.662$ & 0.040 & 0.496 & $0.255-0.967$ \\
\hline
\end{tabular}

Abbreviation: GDFII, growth differentiation factor II.

\section{Enhanced GDFI I expression promoted} apoptosis and downregulated GDFI I expression inhibited apoptosis in

\section{pancreatic cancer cell lines}

To further investigate GDF11's role in pancreatic cancer, we examined the apoptosis rates in aforementioned cell lines with overexpression and knockdown of GDF11. PANC-1 cell line with enhanced GDF11 expression showed significantly higher apoptosis rates (Figure 4A). In accordance with the former result, CFPAC-1 cell line with downregulated GDF11 expression showed significantly lower apoptosis rates (Figure 4B).

\section{Discussion}

Pancreatic cancer is a complex and heterogeneous disease of the digestive system, which is related to various gene deletions, mutations, and amplifications. ${ }^{20}$ Its morbidity and mortality have risen significantly in recent years. ${ }^{3}$ The 5 -year survival rate for pancreatic cancer is one of the worst
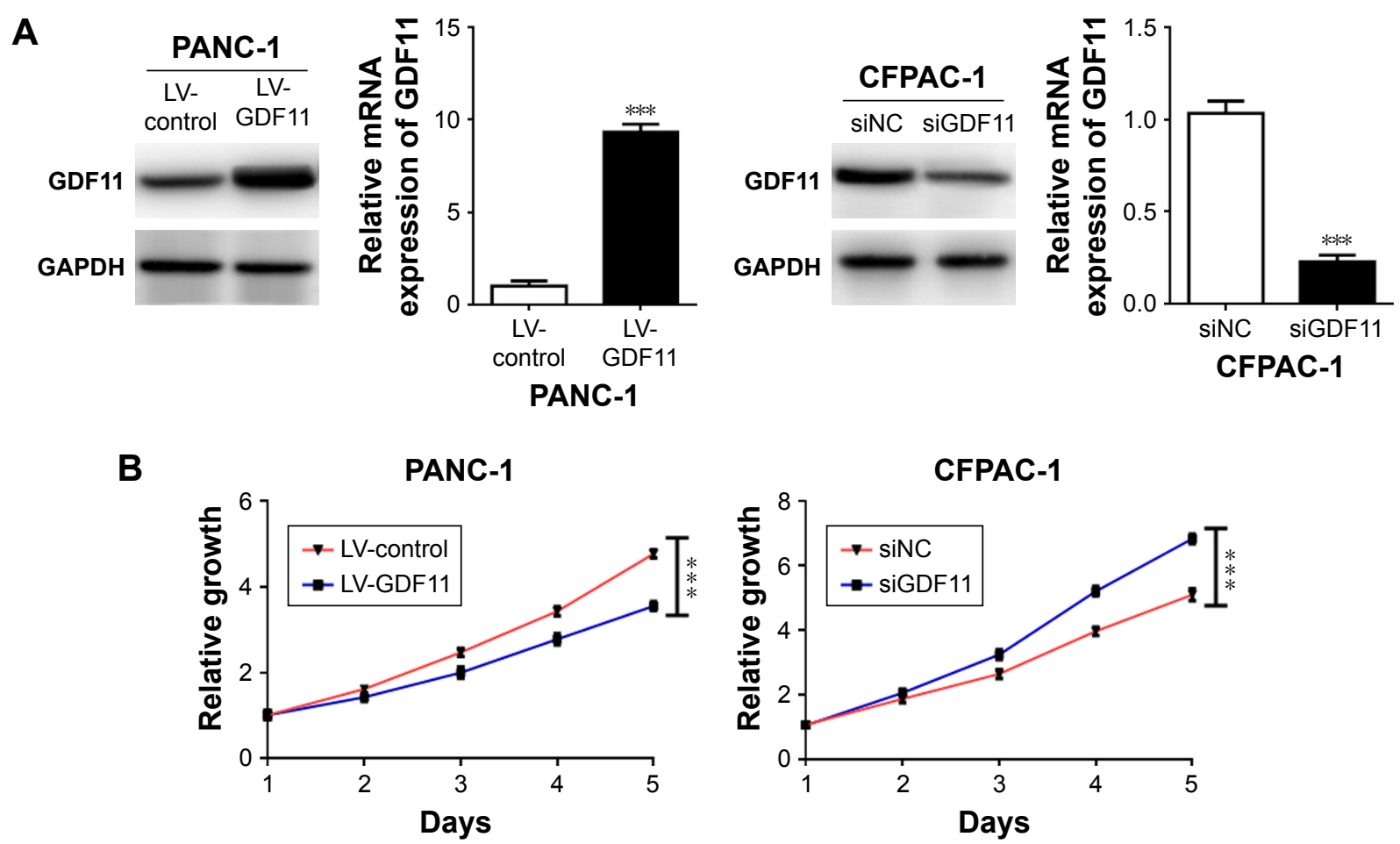

Figure 3 (Continued) 
C
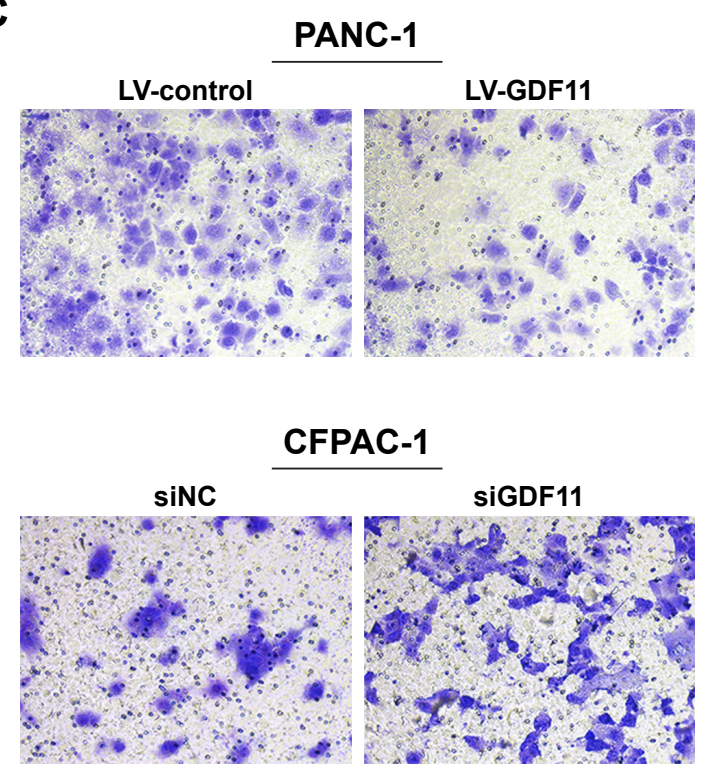

D
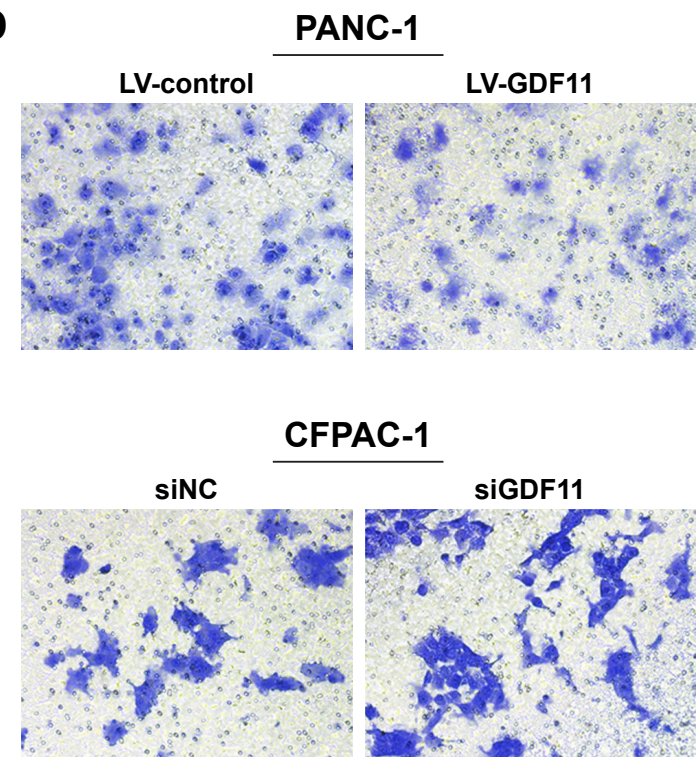
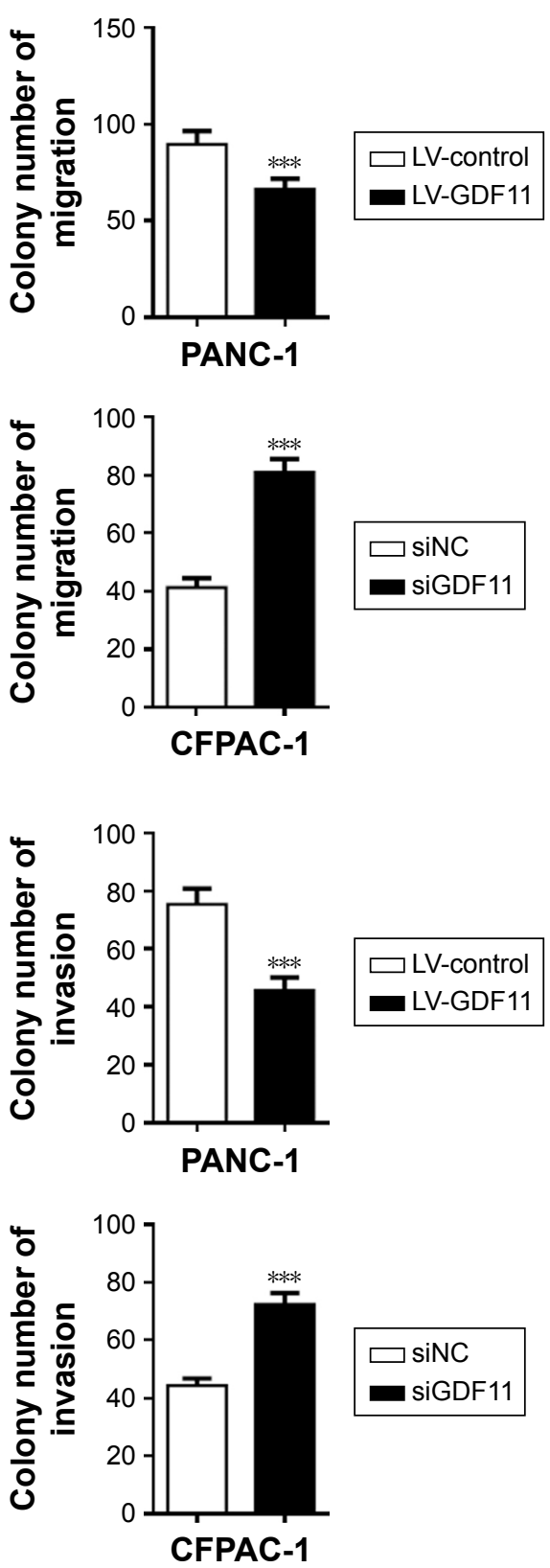

Figure 3 Overexpression of GDFII suppresses tumor growth and metastasis while knockdown of GDFII promoted these functions in pancreatic cancer cell line.

Notes: PANC-I cell was transfected using a lentiviral transduction system to enhance GDFII expression, and CFPAC-I cell was treated using siRNA to inhibit GDFI I expression. (A) Elevated and downregulated GDFII expression was examined by Western blot and qRT-PCR analyses. (B) MTS assay revealed the effects of GDFII overexpression and knockdown on the proliferation of pancreatic cancer cell lines. (C, D) Representative photographs and cell quantifications of transwell migration and invasion assays of elevated and downregulated GDFII in pancreatic cancer cell lines. Data shown represent the mean \pm SD. $* * * P<0.00$ I.

Abbreviations: GDFI I, growth differentiation factor I I; LV, lentiviral vectors; NC, negative control; MTS, 3-(4,5-dimethylthiazol-2-yl)-5-(3-carboxymethoxyphenyl)-2-(4sulfophenyl)-2H-tetrazolium.

prognosis of malignant tumors, at only $5 \% .{ }^{21}$ Risk factors for pancreatic cancer include cigarette smoking, obesity, type 2 diabetes mellitus, alcohol consumption, and chronic pancreatitis. ${ }^{4}$ The early stages of the disease are usually asymptomatic, signifying it is difficult for early diagnosis and treatment. Changing in a series of genes involved in specific pathways and processes contributes to the occurrence and development of pancreatic cancer. However, there are currently no validated biomarkers to predict clinical behavior of this cancer. ${ }^{4}$

In the current study, we first investigated the expression level of GDF11 in tissues and cell lines and then evaluated the relationship between GDF11 protein expression and the clinicopathological features in pancreatic cancer. Results showed that GDF11 expression was significantly lower in pancreatic cancer tissues than that in normal pancreatic tissues. Similar 
A
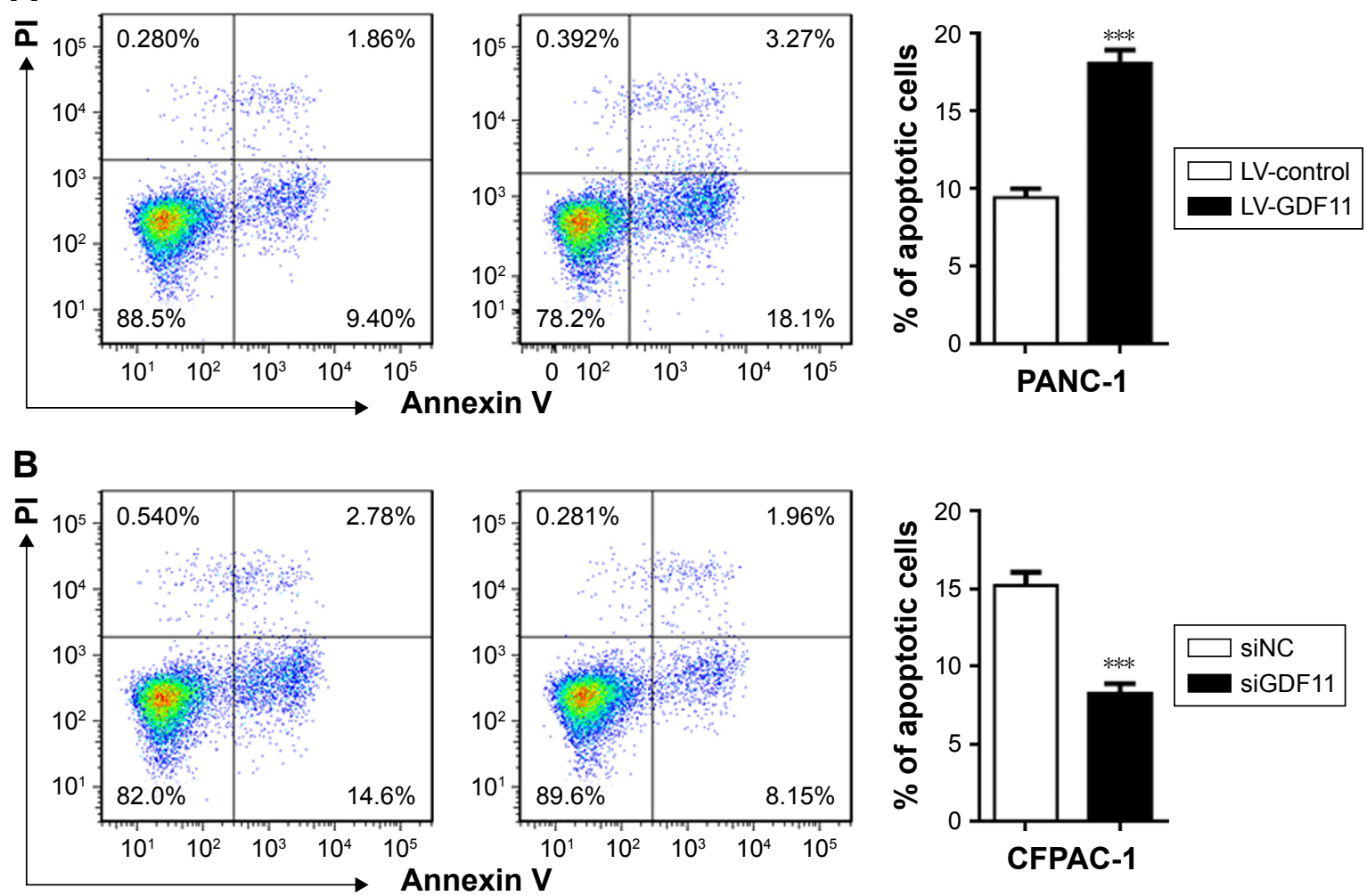

Figure 4 Enhanced GDFI I expression promoted apoptosis and downregulated GDFI I expression inhibited apoptosis in pancreatic cancer cell lines.

Notes: (A) The effect of GDFII overexpression on the apoptosis of pancreatic cancer cell lines by flow cytometry. (B) The effect of knockdown GDFI I on the apoptosis of pancreatic cancer cell line by flow cytometry. Data shown represent the mean \pm SD. $* * * P<0.00 \mathrm{I}$.

Abbreviations: GDFI I, growth differentiation factor II; LV, lentiviral vectors; NC, negative control.

results were demonstrated from the GDF11 expression examination in pancreatic cancer cell lines and human pancreatic duct epithelial cell line. In the cohort of pancreatic cancer patients, high expression of GDF11 in the tumor tissue was found associated with favorable prognosis. Patients with high GDF11 expression showed a significantly higher OS rate. These results are in accordance with prognostic summary of GDF11 in pancreatic cancer in the Human Protein Atlas database. ${ }^{22}$ Moreover, our data demonstrated that high GDF11 protein expression was associated with mild clinical features, such as perineural invasion, T classification, and $\mathrm{N}$ classification. These findings indicated that the high expression of GDF11 may play important roles in the growth, invasion, and metastasis of pancreatic cancer.

Accordingly, we further explored the functional mechanism of GDF11 expression in pancreatic cancer cells. We enhanced GDF11 expression in PANC-1 cell line using a lentiviral transduction system and attenuated GDF11 expression in CFPAC-1 cell line using RNAi treatment. Functional studies indicated that elevated GDF11 expression promoted apoptosis while proliferation, migration, and invasion were suppressed in PANC-1 cell. Instead, inhibited apoptosis, accelerating cell proliferation, migration, and invasion were relevant to lower expression of GDF11 in CFPAC-1 cell. Bokobza et $\mathrm{a}^{23}$ found that GDF9 promoted growth and proliferation of prostate cancer cells by protecting tumor cells from caspase-3-mediated apoptosis. The results of functional experiment showed a tumor-inhibiting effect of GDF11 in pancreatic cancer through restraining tumor growth by promotion of apoptosis.

TGF- $\beta$ superfamily was known as the dual role in cancer. TGF- $\beta$ signaling promotes progression, invasion, and metastasis in advanced cancers while its tumor-suppressing effects were found in normal cells and early cancers. ${ }^{24-26}$ Some reports have revealed the prognostic significance of GDF11 expression in several types of tumors. ${ }^{17,18,27-29}$ Yokoe et al ${ }^{17}$ found that GDF11 mRNA expression in colorectal cancer tissue was significantly higher than in normal tissue. Patients with high GDF11 expression have a high frequency of lymph node metastasis and a significantly poorer OS, indicating that GDF11 might be a novel diagnostic and prognostic biomarker in colorectal cancer patients. However, a recent study showed that GDF11 exerted tumor-suppressing functions in triplenegative breast cancer and its tumor suppression was lost through defective subcellular processing. ${ }^{18}$ In liver cancer, the mRNA and protein expression of GDF11 is downregulated, 
and it plays a tumor-suppressing role through Smad2/3signaling pathway. ${ }^{27}$ The present study suggested that the aberrant expression of GDF11 was significantly correlated with prognosis of pancreatic cancer patients. Kaplan-Meier method showed that patients with low GDF11 expression had worse OS rate than those with high GDF11 expression.

In summary, we demonstrated that GDF11 protein expression significantly decreased in pancreatic cancer tissues and was correlated with tumor progression in pancreatic cancer. The higher expression of GDF11 was significantly correlated with better OS rate of pancreatic cancer patients. Furthermore, overexpression of GDF11 suppressed aggressive behaviors of pancreatic cancer cells may result from its apoptosis-promoting effect on pancreatic cancer cells. Therefore, GDF11 may be a valuable predictive biomarker in assessing the severity and prognosis of pancreatic cancer patients.

\section{Acknowledgments}

This study was approved by the ethics committee of Chinese PLA General Hospital and was carried out in accordance with the Declaration of Helsinki of the World Medical Association. All enrolled patients provided written informed consent.

\section{Disclosure}

The authors declare no conflicts of interest in this work.

\section{References}

1. Chen W, Zheng R, Baade PD, et al. Cancer statistics in China, 2015. CA Cancer J Clin. 2016;66(2):115-132.

2. Hidalgo M. Pancreatic cancer. N Engl J Med. 2010;362(17): 1605-1617.

3. Siegel RL, Miller KD, Jemal A. Cancer statistics, 2016. CA Cancer J Clin. 2016;66(1):7-30.

4. Wolfgang CL, Herman JM, Laheru DA, et al. Recent progress in pancreatic cancer. CA Cancer J Clin. 2013;63(5):318-348.

5. Ansari D, Rosendahl A, Elebro J, Andersson R. Systematic review of immunohistochemical biomarkers to identify prognostic subgroups of patients with pancreatic cancer. Br J Surg. 2011;98(8):1041-1055.

6. Winter JM, Yeo CJ, Brody JR. Diagnostic, prognostic, and predictive biomarkers in pancreatic cancer. J Surg Oncol. 2013;107(1):15-22.

7. Mcpherron AC, Lawler AM, Lee SJ. Regulation of anterior/posterior patterning of the axial skeleton by growth/differentiation factor 11 . Nat Genet. 1999;22(3):260-264.

8. Nickel J, Sebald W, Groppe JC, Mueller TD. Intricacies of BMP receptor assembly. Cytokine Growth Factor Rev. 2009;20(5-6):367-377.
9. Mcpherron AC, Lawler AM, Lee SJ. Regulation of skeletal muscle mass in mice by a new TGF-beta superfamily member. Nature. 1997; 387(6628):83-90.

10. Davis H, Raja E, Miyazono K, Tsubakihara Y, Moustakas A. Mechanisms of action of bone morphogenetic proteins in cancer. Cytokine Growth Factor Rev. 2016;27:81-92.

11. Wakefield LM, Hill CS. Beyond TGF $\beta$ : roles of other TGF $\beta$ superfamily members in cancer. Nat Rev Cancer. 2013;13(5):328-341.

12. Hoshino Y, Katsuno Y, Ehata S, Miyazono K. Autocrine TGF- $\beta$ protects breast cancer cells from apoptosis through reduction of BH3-only protein, Bim. J Biochem. 2011;149(1):55-65.

13. Hu M, Cui F, Liu F, et al. BMP signaling pathways affect differently migration and invasion of esophageal squamous cancer cells. Int $J$ Oncol. 2017;50(1):193-202.

14. Lu X, Jin EJ, Cheng X, et al. Opposing roles of TGF $\beta$ and BMP signaling in prostate cancer development. Genes Dev. 2017;31(23-24): 2337-2342.

15. Huang P, Chen A, He W, et al. BMP-2 induces EMT and breast cancer stemness through Rb and CD44. Cell Death Discov. 2017;3:17039.

16. McPherron AC. Metabolic functions of myostatin and Gdf11. Immunol Endocr Metab Agents Med Chem. 2010;10(4):217-231.

17. Yokoe $\mathrm{T}$, Ohmachi $\mathrm{T}$, Inoue $\mathrm{H}$, et al. Clinical significance of growth differentiation factor 11 in colorectal cancer. Int J Oncol. 2007;31(5): 1097-1101.

18. Bajikar SS, Wang CC, Borten MA, et al. Tumor-suppressor inactivation of GDF11 occurs by precursor sequestration in triple-negative breast cancer. Dev Cell. 2017;43(4):418-435.

19. Shao Y, Li X, Lu Y, Liu L, Zhao P. Aberrant LRP16 protein expression in primary neuroendocrine lung tumors. Int J Clin Exp Pathol. 2015; 8(6):6560-6565.

20. Tan AC, Fan JB, Karikari C, et al. Allele-specific expression in the germline of patients with familial pancreatic cancer: an unbiased approach to cancer gene discovery. Cancer Biol Ther. 2008;7(1):135-144.

21. Krejs GJ. Pancreatic cancer: epidemiology and risk factors. Dig Dis. 2010;28(2):355-358.

22. Zhang Y, Wei Y, Liu D, et al. Role of growth differentiation factor 11 in development, physiology and disease. Oncotarget. 2017;8(46): 81604-81616.

23. Bokobza SM, Ye L, Kynaston HG, Jiang WG. GDF-9 promotes the growth of prostate cancer cells by protecting them from apoptosis. J Cell Physiol. 2010;225(2):529-536.

24. Akhurst RJ, Derynck R. TGF-beta signaling in cancer-a double-edged sword. Trends Cell Biol. 2001;11(11):S44-S51.

25. Lebrun JJ. The dual role of TGF $\beta$ in human cancer: from tumor suppression to cancer metastasis. ISRN Mol Biol. 2012;2012:381428.

26. Massagué J. TGFbeta in cancer. Cell. 2008;134(2):215-230.

27. Zhang YH, Pan LH, Pang Y, et al. GDF11/BMP11 as a novel tumor marker for liver cancer. Exp Ther Med. 2018;15(4):3495-3500.

28. Alvarez C, Aravena A, Tapia T, et al. Different array CGH profiles within hereditary breast cancer tumors associated to BRCA1 expression and overall survival. BMC Cancer. 2016;16:219.

29. Auguściak-Duma A, Sieron AL. Molecular characteristics of leiomyoma uteri based on selected compounds of the extracellular matrix. Postepy Hig Med Dosw. 2008;62:148-165.

\section{Dovepress}

\section{Publish your work in this journal}

OncoTargets and Therapy is an international, peer-reviewed, open access journal focusing on the pathological basis of all cancers, potential targets for therapy and treatment protocols employed to improve the management of cancer patients. The journal also focuses on the impact of management programs and new therapeutic agents and protocols on patient perspectives such as quality of life, adherence and satisfaction. The manuscript management system is completely online and includes a very quick and fair peer-review system, which is all easy to use. Visit http://www.dovepress.com/testimonials.php to read real quotes from published authors. 